\title{
Frequency of Prader-Willi syndrome in births conceived via assisted reproductive technology
}

\author{
June-Anne Gold, MBBS, MRCPCH ${ }^{1,9}$, Chelsey Ruth, MS, $\mathrm{CGC}^{1,9}$, Kathryn Osann, $\mathrm{PhD}^{1}$, \\ Pamela Flodman, MSc, MS1, Barbara McManus², Hye-Seung Lee, PhD³, \\ Sandra Donkervoort, MS, CGC 1,9, Manaswitha Khare, MBBS1, Elizabeth Roof, MA4, \\ Elizabeth Dykens, PhD ${ }^{4}$, Daniel J. Driscoll, MD, PhD ${ }^{5}$, Merlin G. Butler, MD, PhD ${ }^{6,7}$, \\ Janalee Heinemann, MSW2², Suzanne Cassidy, MD² and Virginia E. Kimonis, MD ${ }^{1}$
}

\begin{abstract}
Purpose: Prader-Willi syndrome is an imprinting disorder characterized by typical facial, physical, and cognitive/behavioral features, resulting from lack of paternally expressed genes on chromosome $15 \mathrm{q} 11.2$ q13. Studies have suggested an increased risk of other imprinting disorders in children conceived by assisted reproductive techniques. This study was designed to determine the association between assisted reproductive technology and Prader-Willi syndrome.
\end{abstract}

Methods: Data on individuals with Prader-Willi syndrome were collected from three distinct sources and the proportion of assisted reproductive technology births analyzed.

Results: The proportions of assisted reproductive technology births in the Prader-Willi Syndrome Association (USA), Rare Diseases Clinical Research Network, and University of California, Irvine Medical Center populations were 1.0\% (18/1,736), 1.0\% (1/98), and $2.0 \%(1 / 50)$, respectively (overall $1.1 \%$; population frequency for the
United States was $1.0 \%)$. Of note, $2.4 \%(45 / 1,898)$ of participants were co-twins (11 born after assisted reproductive technology procedures); US twin frequency is $1.6 \%(P=0.007)$. The proportion of individuals with maternal disomy 15/imprinting defects born after assisted reproductive technology was higher than that in the total sample, $55.6 \%(10 / 18)$ and $34.5 \%(431 / 1,250)$, respectively.

Conclusion: This study found no association between assisted reproductive technology and Prader-Willi syndrome. There was an increased frequency of twinning. The number of individuals with maternal disomy 15/imprinting defect was nearly double in the assisted reproductive technology group as compared with the total Prader-Willi syndrome participants.

Genet Med advance online publication 8 August 2013

Key Words: assisted reproductive techniques; imprinting; PraderWilli syndrome; RDCRN; twinning

\section{INTRODUCTION}

Prader-Willi syndrome (PWS) affects $\sim 1$ in 15,000 to 1 in 30,000 individuals ${ }^{1,2}$ and is characterized by typical facial features and major cognitive, behavioral, neurologic, endocrine, and psychiatric issues. PWS is an imprinting disorder caused by three main mechanisms, ultimately resulting in the complete absence of expression of the paternally imprinted genes in the 15q11.2-q13 region. These three genetic mechanisms leading to PWS are paternal deletion of this region (in about $\sim 70 \%$ ), maternal uniparental disomy (in $\sim 25-30 \%$ ), and imprinting center defect (in $\sim 2-5 \%) .{ }^{1-3}$ In the PWS region, the paternal copies of the genes are typically expressed while the maternal copies of these genes are silenced due to parent-of-originspecific imprinting.

PWS is characterized by decreased fetal movement and neonatal hypotonia, decreased activity, and feeding difficulties leading to failure to thrive. Most individuals with PWS have mild intellectual disability, with a mean IQ of $\sim 60-70$, and most display a characteristic behavioral pattern, including temper tantrums, skin-picking, obsessive-compulsive behaviors, stubbornness, and manipulative behavior; attention-deficit and hyperactivity symptoms may also occur, along with features suggestive of autism spectrum disorders. ${ }^{1,4,5}$ Common characteristic facial features of individuals with PWS include almond-shaped and sometimes upslanting palpebral fissures, bitemporal narrowing, and strabismus. ${ }^{1,4}$ Another characteristic feature of PWS in both sexes is hypogonadism, which manifests as genital hypoplasia (including cryptorchidism in males) and delayed or incomplete pubertal development. ${ }^{6}$

The Centers for Disease Control and Prevention (CDC) defines assisted reproductive technology (ART) as fertility treatments involving the handling of both eggs and sperm, not just eggs or

The first two authors contributed equally to this work.

${ }^{1}$ Division of Genetics and Metabolism, Department of Pediatrics, University of California, Irvine, Irvine, California, USA; ${ }^{2}$ Prader-Willi Association (USA), Sarasota, Florida, USA; ${ }^{3}$ Pediatrics Epidemiology Center, University of South Florida, Tampa, Florida, USA; ${ }^{4}$ Vanderbilt Kennedy Center, Vanderbilt University, Nashville, Tennessee, USA; ${ }^{5}$ Department of Pediatrics, University of Florida, Gainesville, Florida, USA; ${ }^{6}$ Department of Psychiatry and Behavioral Sciences, University of Kansas Medical Center, Kansas City, Kansas, USA; ${ }^{7}$ Department of Pediatrics, University of Kansas Medical Center, Kansas City, Kansas, USA; ${ }^{8}$ Division of Medical Genetics, Department of Pediatrics, University of California, San Francisco, San Francisco, California, USA. ${ }^{9}$ Present address: Division of Medical Genetics and Metabolism, Department Pediatrics, Loma Linda University Medical Center, Loma Linda, California, USA (J.-A.G.); Fetal Diagnostic Center, Kapiolani Medical Center for Women and Children, Honolulu, Hawaii, USA (C.R.); Neuromuscular and Neurogenetic Disorders of Childhood Section, Neurogenetics Branch, National Institute of Neurological Disorders and Stroke, National Institutes of Health, Bethesda, Maryland, USA (S.D.). Correspondence: June-Anne Gold (jgold@llu.edu) or Virginia E. Kimonis (vkimonis@uci.edu) 
sperm. Such procedures include in vitro fertilization (IVF), intracytoplasmic sperm injection (ICSI), gamete intrafallopian transfer (GIFT), and zygote intrafallopian transfer (ZIFT). According to the CDC and the National Center for Health Statistics, the 2006 US frequency of ART births, including only IVF, ICSI, GIFT, and ZIFT procedures, was $<1.0 \%{ }^{7}$ Other methods, such as intrauterine insemination or fertility drugs to induce ovarian stimulation or ovulation, can also be classified as ART; however, they are not included in the CDC definition.

\section{Imprinting disorders and ART}

Recent reports have provided evidence for a relationship between imprinting disorders and assisted reproductive technologies. Angelman and Beckwith-Wiedemann syndromes are two disorders in which an imprinting defect accounts for a significant proportion of affected cases, with known increased risks for children born after ART. ${ }^{8-10}$ An association between PWS and ART has not been previously identified. It has been hypothesized that because maternal uniparental disomy (UPD) and paternal deletions account for the majority of PWS cases, PWS may not be associated with ART. ${ }^{9}$ Nevertheless, previous studies in Europe investigating this possible relationship have been conducted with small sample sizes and thus failed to ascertain a large number of individuals with PWS. To date, our study represents the largest one in the United States investigating the association between this imprinting disorder and ART.

\section{MATERIALS AND METHODS}

This study followed unique data collection protocols for three distinct PWS populations including the following:

\section{The Prader-Willi Syndrome Association (USA)}

A survey about prenatal, medical, and family histories was created and posted on the organization's website in 2004. Families with an individual with PWS who were members of PraderWilli Syndrome Association (PWSA, USA) were invited to participate in the survey; 1,600 such families responded to questionnaire 1 regarding the use of assisted reproductive techniques for conception of the individuals with PWS. Those families who underwent ART were invited to complete a secondary survey, referred to as questionnaire 2, for our study. Questionnaire 2 included repeated questions from the first PWSA (USA) survey to confirm initial responses. These repeated questions were answered the same in both questionnaires. Questionnaire 2 requested the following information about the individual with PWS:

- Maternal and paternal ages at participant's birth,

- Genetic subtype of PWS,

- Type of ART utilized,

- Location where the ART procedure was performed,

- Number, ages, and sex of other siblings,

- Parents' history of miscarriages, and

- Whether the participant was a co-twin.
For this study, data collected from questionnaire 1 included age, birth year, and sex. Data were also collected on whether an individual with PWS was born following ART.

\section{The Rare Diseases Clinical Research Network}

Data on individuals with PWS were collected through the use of the National Institutes of Health-sponsored Rare Diseases Clinical Research Network (RDCRN) Natural History PWS and Morbid Obesity Clinical Protocol (institutional review board protocol 2007-5605). This RDCRN study is composed of four centers, including the University of Florida Health Science Center in Gainesville, Florida; University of Kansas Medical Center in Kansas City, Kansas; Vanderbilt University Medical Center in Nashville, Tennessee; and Baylor College of Medicine in Houston, Texas.

At the time of the study, the RDCRN database contained a total of 108 participants enrolled at the four centers. Demographic, medical, educational, and familial surveys were completed for each individual in the database. In addition, the following data were collected from the Data Technology Coordinating Center on behalf of the RDCRN: age, birth year, sex, PWS genetic subtype, maternal age at birth, and paternal age at birth.

\section{University of California, Irvine Medical Center Clinical Service}

Information about individuals with PWS who were seen by the University of California, Irvine Medical Center (UCIMC) Division of Genetics and Metabolism is maintained in the local database. Medical records for each individual were reviewed to confirm the diagnosis, determine the genetic PWS subtype, and investigate whether conception included the use of assisted reproductive techniques. Other data collected from the medical records were the age, birth year, sex, maternal age at birth, and paternal age at birth.

In the United States, all clinics that perform ART procedures must report their pregnancy success rate data to the CDC under the Fertility Clinic Success Rate and Certification Act of 1992. The Society for Assisted Reproductive Technology maintains a database of ART procedures performed in these clinics each year and makes these data available to the CDC. The CDC publishes these results and estimates that the database accounts for $\sim 95 \%$ of ART procedures performed in the United States each year. These data were used to determine the incidence of ART births in the United States to compare the incidence of ART births in the PWS study population during similar birthyears. ${ }^{7}$ Only procedures defined by the CDC, including IVF, ICSI, GIFT, and ZIFT, were considered ART.

This study involved review of medical records and questionnaire responses from participants at the three distinct clinical sites to investigate the proportion of ART births in the PWS study population and the proportion of the two genetic mechanisms causing PWS, maternal UPD and imprinting defects, in the ART-conceived and naturally conceived groups. The sample sizes for the RDCRN and UCIMC in particular were limited. 
Statistical analysis was performed using SPSS for Windows software (version 15.0; SPSS, Chicago, IL). To compare the twin proportion or ART-birth proportion in the PWS study population with that in the general US population, Pearson $\chi^{2}$ test (one-sample goodness-of-fit) was used; $P<0.05$ was considered to be statistically significant. Comparisons among the various proportions of genetic subtypes were performed by testing the marginal frequencies of the contingency tables for these three independent clinic sites.

\section{RESULTS}

Of the total 1,898 individuals with PWS surveyed for this study, 20 were conceived after ART procedures defined by the CDC (18 from the PWSA (USA) and one each from the RDCRN and UCIMC). The mean ages at the time of this study for the individuals with PWS from PWSA (USA), RDCRN, and UCIMC were 19.0, 10.1, and 15.0 years, respectively. The range of birth-years for those individuals with PWS born after ART was from 1994 to 2006. The combined proportion was $1.1 \%(20 / 1,884$; $95 \%$ confidence interval (CI): $0.6-1.6 \%)$. The number and percentage of those conceived after ART are displayed in Table 1.

The CDC has been publishing the estimated US rate of individuals born after IVF, ICSI, GIFT, and ZIFT procedures annually since 1995 . No other ART procedures were included in this estimation. To compare accurately the proportion of ART births in the PWS study population with that in the general US population, only those conceived after IVF, ICSI, GIFT, or ZIFT procedures were included as ART births for this comparison (Table 2). Twenty participants were conceived after CDC-recognized ART procedures (IVF, ICSI, and ZIFT; none were reported as conceived following GIFT). Twenty participants conceived following intrauterine insemination or ovarian stimulation, both non-CDC ART procedures, were included in the naturally conceived group.

The proportion of ART births in the PWS study population (including only the 20 conceived after IVF, ICSI, or ZIFT procedures) was $1.1 \%$ (20/1,884; 95\% CI: 0.6-1.6\%). The ART status for 10 RDCRN participants was unknown, so these individuals were excluded from the study. Four PWSA (USA) participants

Table 1 Number and percentage of ART-conceived participants with PWS from each studied group

\begin{tabular}{lcccc} 
& PWSA (USA) & RDCRN & UCIMC & Total \\
\cline { 2 - 5 } & $\boldsymbol{n ( \% )}$ & $\boldsymbol{n ( \% )}$ & $\boldsymbol{n ( \% )}$ & $\boldsymbol{n ( \% )}$ \\
\hline $\begin{array}{l}\text { ART- } \\
\text { conceived }\end{array}$ & $18(1.0)$ & $1(1.0)$ & $1(2.0)$ & $20(1.1)$ \\
$\begin{array}{l}\text { Naturally } \\
\text { conceived }\end{array}$ & $1,718(99.0)$ & $97(99.0)$ & $49(98.0)$ & $1,864(98.9)$ \\
Total & $1,736(100.0)$ & $98(100.0)$ & $50(100.0)$ & $1,884(100.0)$ \\
$\begin{array}{l}\text { Unknown } \\
\text { ART use }\end{array}$ & 4 & 10 & 0 & 14 \\
\hline
\end{tabular}

$\chi^{2}=0.072 ; 1 \mathrm{df} ; P=0.788$.

ART, assisted reproductive technology; PWS, Prader-Willi syndrome; PWSA, Prader-Willi Syndrome Association; RDCRN, Rare Diseases Clinical Research Network; UCIMC, University of California, Irvine Medical Center. were also excluded from the study because they did not provide the specific type of ART utilized. These omitted data did not significantly change the study's overall proportion of ART births of $1.1 \%$. The mean frequency of US ART births during the 12 years in which the study participants were born was calculated to be $1.0 \%$. The proportions of ART births in the PWS study population and the general US population were calculated to be similar by Pearson $\chi^{2}$ test $(P=0.788)$, indicating that there was no significant increase in ART births in the PWS study population.

The mean parental ages in the ART-conceived group may provide an explanation for the increased proportion of maternal UPD/imprinting defects seen in the ART-conceived group. However, data on parental ages were available for only a small number of naturally conceived participants and an even smaller number of ART conceived participants from each site. Statistical comparison on mean parental ages for the ART-conceived and naturally conceived groups from the RDCRN and UCIMC sites could not be performed separately because of the small sample size and limited power. For example, there were only two participants (one ART conceived and one naturally conceived) from the RDCRN with parental ages reported. There was also a strong imbalance in the number of participants with parental ages in the UCIMC ART and non-ART groups: maternal ages were available for one ART-conceived patient and 49 naturally conceived patients, whereas paternal ages were available for one ART-conceived patient and 48 naturally conceived patients. Therefore, statistical comparison of mean parental ages for the ART-conceived and naturally conceived groups was restricted to the PWSA (USA) site only.

From the PWSA (USA) site, 18 participants reported maternal ages (10 ART conceived and 8 naturally conceived), and 16 reported paternal ages (9 ART conceived and 7 naturally conceived). Although PWSA (USA) also had a small sample size, the number of participants with parental ages was fairly consistent between the ART and non-ART groups. The mean parental ages at birth were greater in the ART-conceived group (36.3 and 39.7 years, respectively, for mothers and fathers) in comparison

Table 2 Number and percentage of ART-conceived participants with PWS from each studied group with each ART procedure

\begin{tabular}{lclll} 
& PWSA (USA) & RDCRN & UCIMC & Total \\
\cline { 2 - 5 } Type of ART & $\boldsymbol{n ( \% )}$ & $\boldsymbol{n ( \% )}$ & $\boldsymbol{n}(\%)$ & $\boldsymbol{n}(\%)$ \\
\hline IVF variations & & & & \\
$\quad$ IVF only & $9(50.0)$ & $0(0)$ & $1(100)$ & $10(50.0)$ \\
IVF/ICSI & $3(16.7)$ & $0(0)$ & $0(0)$ & $3(15.0)$ \\
IVF/donor egg & $5(27.8)$ & $1(100)$ & $0(0)$ & $6(30.0)$ \\
ZIFT & $1(5.5)$ & $0(0)$ & $0(0)$ & $1(5.0)$ \\
Total & $18(100)$ & $1(100)$ & $1(100)$ & $20(100)$ \\
\hline
\end{tabular}

ART, assisted reproductive technology; ICSI, intracytoplasmic sperm injection; IVF, in vitro fertilization; PWS, Prader-Willi syndrome; PWSA, Prader-Willi Syndrome Association; RDCRN, Rare Diseases Clinical Research Network; UCIMC, University of California, Irvine Medical Center; ZIFT, zygote intrafallopian transfer. 
with the naturally conceived group ( 31.5 and 31.6 years, respectively). These differences in ART and non-ART parental ages were statistically significant $(P=0.049$ and $P=0.020$ for mothers and fathers, respectively).

\section{Frequency of the genetic subtypes of PWS}

This study hypothesized a higher proportion of maternal UPD and imprinting center defects in ART-conceived individuals as compared with those conceived naturally. Table 3 reports the data for all participants for whom the genetic subtype was available $(N=1,250)$, by clinic site. Those with maternal UPD and imprinting defects were combined because the two genetic subtypes were not distinguished for some participants. For example, 333 and 37 participants from PWSA (USA) had maternal UPD and imprinting defects, respectively. The RDCRN data included 32 with maternal UPD, 4 with imprinting defects, and 3 with either maternal UPD or imprinting defects. The UCIMC data included 9 with maternal UPD and 13 with either maternal UPD or imprinting defects. These data were combined across the three sites.

These data were compared with the expected proportion of the genetic subtypes in the general PWS population. The observed proportion of participants with a deletion was $65.5 \%$ (819/1,250; 95\% CI: 62.9-68.1\%), which is consistent with the expected range of $65-75 \%$. The observed proportion with maternal UPD and imprinting defects was 34.5\% (431/1,250; 95\% CI: 31.9-37.1\%), consistent with the expected range of $25-35 \%$.

The two genetic subgroups were found in different proportions in the ART-conceived group as compared with those conceived naturally $(P=0.02)$. In the ART-conceived group, the proportion with maternal UPD or imprinting defects was $55.6 \%(10 / 18)$, whereas among those conceived naturally, the proportion was $34.2 \%(421 / 1,232)$. As compared with naturally conceived participants, those who were ART conceived were more likely to have UPD and imprinting defects than deletions.

\section{Frequency of twins}

The number of participants who were co-twins was evaluated to determine the frequency of twinning in the PWS population with or without the use of ART procedures. Table 4 displays the number of co-twins from each site; only one twin of each twin pair was affected with PWS. There was no significant difference in the proportion of twins from each site; therefore, these data were combined to calculate the total twin frequency for this study. The total proportion of twins of $2.4 \%(45 / 1,898$; $95 \%$ CI: $1.7-3.1 \%)$ was statistically significantly different $(P=$ 0.007 ) from the US twin frequency of $1.6 \%{ }^{7}$ Because previous studies reported an increased rate of twinning (both monozygotic and dizygotic) after the use of assisted reproduction, ${ }^{11-13}$ 11 (24.4\%) of these PWS co-twins, all fraternal, who were conceived after ART procedures, were excluded from the overall twin frequency in the PWS study population. For all naturally conceived PWS patients, a modified twin frequency of $1.8 \%$ (34/1,844; 95\% CI: $1.2-2.4 \%)$ was obtained, which was not significantly different from the US population twin frequency $(P=0.404)$. Thirty-one percent $(14 / 45)$ of the total PWS cotwins were reported as identical twins, and $69 \%(31 / 45)$ were reported as fraternal twins.

Table 3 Number and percentage of naturally conceived and ART-conceived participants with PWS from each site with a deletion or maternal UPD/imprinting defect

\begin{tabular}{|c|c|c|c|c|c|c|c|c|}
\hline \multirow[b]{3}{*}{$\begin{array}{c}\text { Genetic subtype } \\
\text { of PWS }\end{array}$} & \multicolumn{2}{|c|}{ PWSA (USA) } & \multirow{2}{*}{\multicolumn{2}{|c|}{$\begin{array}{c}\text { RDCRN } \\
n(\%)\end{array}$}} & \multirow{2}{*}{\multicolumn{2}{|c|}{$\begin{array}{c}\text { UCIMC } \\
n(\%)\end{array}$}} & \multirow{2}{*}{\multicolumn{2}{|c|}{$\begin{array}{l}\text { Total } \\
n(\%)\end{array}$}} \\
\hline & & & & & & & & \\
\hline & $\begin{array}{l}\text { Naturally } \\
\text { conceived }\end{array}$ & $\begin{array}{c}\text { ART } \\
\text { conceived }\end{array}$ & $\begin{array}{l}\text { Naturally } \\
\text { conceived }\end{array}$ & $\begin{array}{c}\text { ART } \\
\text { conceived }\end{array}$ & $\begin{array}{l}\text { Naturally } \\
\text { conceived }\end{array}$ & $\begin{array}{c}\text { ART } \\
\text { conceived }\end{array}$ & $\begin{array}{l}\text { Naturally } \\
\text { conceived }\end{array}$ & $\begin{array}{c}\text { ART } \\
\text { conceived }\end{array}$ \\
\hline Deletion & $726(66.8)$ & $7(43.7)$ & $62(61.4)$ & $1(100.0)$ & $23(52.3)$ & $0(0)$ & $811(65.8)$ & $8(44.4)$ \\
\hline $\begin{array}{l}\text { Maternal UPD/ } \\
\text { imprinting defect }\end{array}$ & $361(33.2)$ & 9 (56.3) & 39 (38.6) & $0(0)$ & $21(47.7)$ & $1(100.0)$ & $421(34.2)$ & $10(55.6)$ \\
\hline
\end{tabular}

ART, assisted reproductive technology; PWS, Prader-Willi syndrome; PWSA, Prader-Willi Syndrome Association; RDCRN, Rare Diseases Clinical Research Network; UCIMC, University of California, Irvine Medical Center; UPD, uniparental disomy.

Table 4 Number and percentage of participants with PWS who were co-twins

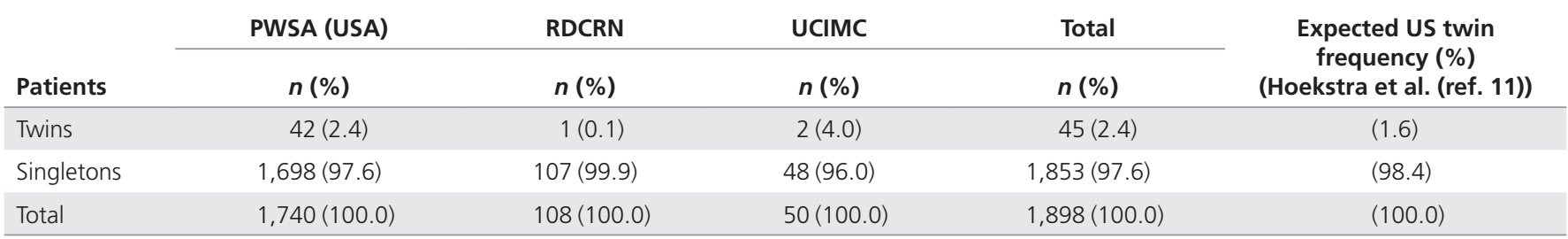

$\chi^{2}=7.162 ; 1 \mathrm{df} ; P=0.007$.

PWS, Prader-Willi syndrome; PWSA, Prader-Willi Syndrome Association; RDCRN, Rare Diseases Clinical Research Network; UCIMC, University of California, Irvine Medical Center. 


\section{DISCUSSION}

This study demonstrated that the proportion of ART births in the PWS study population was not significantly increased above that in the general US population. Results from this study were consistent with previous research, suggesting that ART is not associated with an increased risk of PWS.

Multiple studies have concluded that the effects of ART procedures may be restricted to imprinting disorders, such as Angelman syndrome and Beckwith-Wiedemann syndrome, in which methylation patterns result in the loss of expression of the maternal allele, or in which an imprinting defect accounts for a significant proportion of affected cases. ${ }^{14,15}$ An imprinting center defect in an ART-conceived individual with PWS has not been previously reported in the literature; ${ }^{9,16}$ however, two ART-conceived individuals confirmed with imprinting center defects were found in our study.

Although this study did not find a significantly increased proportion of ART births in the PWS study population using the CDC definition (including IVF, ICSI, and ZIFT), the sample also includes individuals who were conceived using other ART methods, such as ovulation-inducing drugs and intrauterine insemination. The use of these procedures should not be ignored in this PWS population because they may contribute to an increased frequency of imprinting disorders following all ART techniques. ${ }^{17}$ Therefore, it is imperative to note all types of assisted reproductive techniques utilized in order to further investigate their effects on imprinting mechanisms and association with PWS.

These results support a significant difference between the ART-conceived and naturally conceived groups with respect to the proportion of maternal UPD and imprinting center defects. However, overall, there was no significant association detected between ART and PWS. One limitation to this comparison was the small sample size; data on the PWS genetic subtypes were available for only 18 of the 20 participants in the CDC-defined ART group.

The mean parental ages in the ART-conceived group may provide an explanation for the increased proportion of maternal UPD/imprinting defects seen in the ART-conceived group. Statistical comparisons were performed for the PWSA (USA) group only, and the mean maternal age at birth was greater in the ART-conceived group ( 36.3 years) than in the naturally conceived group (31.5 years) $(P=0.049)$. The mean paternal age at birth was also greater in the ART-conceived group (39.7 years) than in the non-ART conceived group (31.6 years) $(P=0.020)$. This statistical comparison was limited by the small sample sizes and low power. Parental data from the RDCRN and UCIMC groups could not be compared separately within each group nor could it be combined with the UCIMC parental data.

However, these results are consistent with previous studies looking at the maternal age effects on the increased incidence of maternal UPD in individuals with PWS. Matsubara et al. ${ }^{18}$ studied maternal age effect on the development of PWS in 117 Japanese patients with PWS. Their results implied that the advanced maternal age at childbirth is a predisposing factor for the development of maternal 15 UPD because of increased meiosis 1 errors.

Moreover, Whittington et al. reported a greater proportion (50\%) of maternal UPD in 34 individuals with PWS who were younger than 5 years. Whittington et al. ${ }^{19,20}$ proposed that increased maternal age at the individual's birth would most likely explain the changing proportions of UPD and deletions in this generation of individuals with PWS. The possible differences in parental ages in the ART-conceived and naturally conceived individuals with PWS may also suggest an explanation for the observed ART-birth proportion of $2.3 \%(44 / 1,888)$ when all types of assisted reproduction (not just IVF, ICSI, GIFT, and ZIFT) are considered. The individuals in the ART-conceived group may be more likely to have older mothers and fathers. These older parents may have experienced infertility issues due to advanced parental ages and pursued ART procedures to conceive a child. As previously mentioned, maternal UPD is associated with increasing maternal age, so the ART-conceived participants may be affected with PWS due to mechanisms causing UPD and not due to the ART procedures themselves.

Another possible explanation for the increased proportion of maternal UPD and imprinting defects found in the ARTconceived PWS population may be a response bias, and more individuals with maternal UPD may have been included in this study. For example, families with a child with PWS with maternal UPD may be more involved in the PWS community. Individuals with PWS due to maternal UPD are less severely affected; they have higher verbal IQ and milder physical features than those with deletions. ${ }^{1,5,21}$ The milder PWS phenotype in younger individuals with UPD may allow families to be more involved in studies and to participate in questionnaires. If this is the case, the data may be biased, with fewer individuals with a paternal deletion included in this study.

Response bias could have also occurred across sites because each study group utilized different methods to collect data from patients. The PWSA (USA) used voluntary and anonymous questionnaires, with the initial survey posted online. The RDCRN collected data strictly on a research basis through its network, whereas data from the UCIMC patients were obtained from a clinical perspective during genetics visits. Completing an online questionnaire may have been more convenient for some families instead of scheduling face-to-face genetics appointments. In any case, reporting bias could not be ruled out. Finally, the stigma associated with infertility could have contributed to less-than-honest reporting of ART for some families; however, this particular response bias could have been applied to all three sites.

Although this study agrees with previous reports stating no association between ART and PWS, ${ }^{22}$ further investigation with a larger sample size and higher power is needed to detect a possible association. Furthermore, it still remains to be determined whether other factors besides IVF and ICSI procedures are affecting the increased incidence of imprinting disorders. ${ }^{23}$ Previous studies have reported that imprinting defects may be caused by either the subfertility of the couple or superovulation 
and hormone treatment and are not due to the ART procedure itself. For instance, Ludwig et al. ${ }^{17}$ reported an increased prevalence of imprinting defects in patients with Angelman syndrome born to subfertile couples and suggested that superovulation, rather than ICSI, may further increase the risk of an imprinting defect in the child. Sutcliffe et al. ${ }^{9}$ also discovered similar results. In addition, some of the imprinting disturbances suggested to be associated with ART may already be present in the gametes of infertile men being used for ICSI. ${ }^{24}$

Most evidence related to ART suggests altered methylation status of the maternal allele, or the female gamete imprint. This maternal imprint is erased and reestablished in female gametes before ovulation at the $1 \mathrm{n}$ stage, which occurs much later in comparison to the establishment of the paternal imprint at the $2 \mathrm{n}$ stage in spermatogenesis. ${ }^{25}$ The critical time difference in gametogenesis for the maintenance of parental imprints supports the hypothesis that ART can affect methylation of maternal imprints rather than paternal imprints. This also supports the finding that PWS is not increased after ART, given that the molecular cause of this condition is the loss of the paternal allele, which is not methylated. Future research investigating these hypotheses must be performed to elucidate true causes for the increased incidence of imprinting disorders in children born after ART procedures.

This study also demonstrated no association between twinning and PWS when ART-conceived pregnancies were excluded.

\section{Conclusion}

This study did not find an increased risk of ART-conceived pregnancies among individuals with PWS but did find a significantly increased proportion of maternal UPD and imprinting defects in the ART-conceived PWS study population. Although this finding cannot provide a definite link between ART and increased frequencies of maternal UPD and imprinting defects, it is anticipated that the data collected will provide a foundation for future investigations. Results from long-term studies are also necessary to properly counsel couples considering these assisted reproductive technologies and may possibly suggest the need to offer genetic screening, evaluation, and monitoring of ART-conceived children.

Although the absolute risk of PWS, and other imprinting diseases, may be small with ART, further investigations, including large, long-term prospective studies, are recommended to assess the health and development of ART-conceived children.

\section{ACKNOWLEDGMENTS}

We thank the individuals and families who have contributed to this work and their health-care providers. This study was supported by National Institutes of Health U54 grants RR019478 and HD061222, Office of Rare Diseases Research. The authors acknowledge the gracious participation and provision of information by the families of the reported participants. Mary Lou Oster-Granite, health scientist administrator at NICHD, provided invaluable guidance, support, and encouragement for this rare disease initiative. We thank the Institute for Clinical and Translational Science (ICTS) at the University of Kansas Medical Center, Kansas City, KS; University of Florida, Gainesville, FL; Vanderbilt Kennedy Center, Vanderbilt University, Nashville, TN; and the University of California, Irvine, Irvine, CA.

\section{DISCLOSURE}

The authors declare no conflict of interest.

\section{REFERENCES}

1. Cassidy SB, Driscoll DJ. Prader-Willi syndrome. Eur J Hum Genet 2009;17:3-13.

2. Butler MG. Prader-Willi Syndrome: obesity due to Genomic Imprinting. Curr Genomics 2011;12:204-215.

3. Horsthemke $B$, Wagstaff J. Mechanisms of imprinting of the Prader-Willi/ Angelman region. Am J Med Genet A 2008;146A:2041-2052.

4. Whittington J, Holland T. Prader-Willi Syndrome: Development and Manifestations. Cambridge University Press: Cambridge, UK, 2004.

5. Butler M, Lee, PDK, Whitman, BY. Management of Obesity in Prader-Willi Syndrome, 3rd edn. Springer-Verlag: New York, 2006.

6. Gunay-Aygun M, Schwartz S, Heeger S, O'Riordan MA, Cassidy SB. The changing purpose of Prader-Willi syndrome clinical diagnostic criteria and proposed revised criteria. Pediatrics 2001;108:E92.

7. Centers for Disease Control and Prevention. Assisted reproductive technology. (2009). (http://www.cdc.gov/art/ART2009/PDF/ART_2009_Full.pdf.)

8. Maher ER. Imprinting and assisted reproductive technology. Hum Mol Genet 2005;14 Spec No 1:R133-R138.

9. Sutcliffe AG, Peters CJ, Bowdin S, et al. Assisted reproductive therapies and imprinting disorders-a preliminary British survey. Hum Reprod 2006;21:10091011.

10. Cox GF, Bürger J, Lip V, et al. Intracytoplasmic sperm injection may increase the risk of imprinting defects. Am J Hum Genet 2002;71:162-164.

11. Hoekstra C, Willemsen G, van Beijsterveldt TC, Montgomery GW, Boomsma DI. Familial twinning and fertility in Dutch mothers of twins. Am J Med Genet $A$ 2008;146A:3147-3156.

12. Vitthala S, Gelbaya TA, Brison DR, Fitzgerald CT, Nardo LG. The risk of monozygotic twins after assisted reproductive technology: a systematic review and meta-analysis. Hum Reprod Update 2009;15:45-55.

13. Aston $\mathrm{KI}$, Peterson CM, Carrell DT. Monozygotic twinning associated with assisted reproductive technologies: a review. Reproduction 2008;136:377-386.

14. Arnaud P, Feil R. Epigenetic deregulation of genomic imprinting in human disorders and following assisted reproduction. Birth Defects Res C Embryo Today 2005;75:81-97.

15. Amor DJ, Halliday J. A review of known imprinting syndromes and their association with assisted reproduction technologies. Hum Reprod 2008:23:2826-2834.

16. Doornbos ME, Maas SM, McDonnell J, Vermeiden JP, Hennekam RC. Infertility, assisted reproduction technologies and imprinting disturbances: a Dutch study. Hum Reprod 2007;22:2476-2480.

17. Ludwig M, Katalinic A, Gross S, Sutcliffe A, Varon R, Horsthemke B. Increased prevalence of imprinting defects in patients with Angelman syndrome born to subfertile couples. J Med Genet 2005;42:289-291.

18. Matsubara K, Murakami N, Nagai T, Ogata T. Maternal age effect on the development of Prader-Willi syndrome resulting from upd(15)mat through meiosis 1 errors. J Hum Genet 2011;56:566-571.

19. Whittington JE, Butler JV, Holland AJ. Changing rates of genetic subtypes of Prader-Willi syndrome in the UK. Eur J Hum Genet 2007;15:127-130.

20. Butler MG, Sturich J, Myers SE, Gold JA, Kimonis V, Driscoll DJ. Is gestation in Prader-Willi syndrome affected by the genetic subtype? J Assist Reprod Genet 2009;26:461-466.

21. Roof E, Stone W, MacLean W, Feurer ID, Thompson T, Butler MG. Intellectual characteristics of Prader-Willi syndrome: comparison of genetic subtypes. J Intellect Disabil Res 2000;44 (Pt 1):25-30.

22. Neri QV, Takeuchi T, Palermo GD. An update of assisted reproductive technologies results in the United States. Ann N Y Acad Sci 2008;1127:41-48.

23. Manipalviratn S, DeCherney A, Segars J. Imprinting disorders and assisted reproductive technology. Fertil Steril 2009;91:305-315.

24. Marques CJ, Costa P, Vaz B, et al. Abnormal methylation of imprinted genes in human sperm is associated with oligozoospermia. Mol Hum Reprod 2008;14:67-74

25. Owen CM, Segars JH Jr. Imprinting disorders and assisted reproductive technology. Semin Reprod Med 2009;27:417-428. 